\title{
Atmospheric Pressure Plasma Iradiation on Embryonic Stem Cells: Signals and Differentiation
}

\author{
Shoko Nishihara, ${ }^{*}$ Hayato Ota, \& Taichi Miura \\ Laboratory of Cell Biology, Department of Bioinformatics, Graduate School of Engineering, Soka \\ University, Tokyo 192-8577, Japan \\ *Address all correspondence to: Shoko Nishihara, 1-236 Tangi-machi, Hachioji, Tokyo 192-8577, Japan; Tel.: 81-426- \\ 91-8140, E-mail: shoko@soka.ac.jp
}

\begin{abstract}
Mouse embryonic stem cells (ESCs) are pluripotent stem cells (PSCs) derived from pre-implantation embryos. They possess self-renewal properties in vitro and can differentiate into various types of cells. Signaling via extrinsic factors is one of the main factors that determine cell fate during the differentiation of PSCs. For example, fibroblast growth factor (FGF) signal enhances the differentiation of ESCs into ectoderm and neural stem cells in mice and in humans. Recently, reactive oxygen species (ROS) have been reported to regulate various signaling pathways. A large number of reactive species such as ROS and reactive nitrogen species are generated by atmospheric pressure plasma (APP) irradiation. We analyzed the effects of APP irradiation on the maintenance and differentiation of mouse ESCs, focusing on signal control. We found that APP irradiation inhibited the proliferation of mouse ESCs, but did not affect their undifferentiated state. In contrast, APP irradiation did not inhibit the proliferation of embryoid bodies, but did inhibit the differentiation of mouse ESCs to mesoderm and endoderm by inhibiting Wnt signal. APP irradiation enhanced the differentiation to the epiblast, ectoderm, and neuronal lineages by activation of FGF4 signaling. This suggests that the latter effects are caused by the $\mathrm{H}_{2} \mathrm{O}_{2}$ generated in the medium by APP irradiation.
\end{abstract}

KEY WORDS: atmospheric pressure plasma, embryonic stem cells, ES cells, differentiation, signal pathways, hydrogen peroxide, ROS, embryoid body formation, fibroblast growth factor 4, Wnt

\section{INTRODUCTION}

Embryonic stem cells (ESCs), which possess pluripotency and self-renewal properties, are originally derived from the inner cell mass of blastocysts. They can differentiate into all types of the cells constituting the adult body. The first ESC lines were established from mouse pre-implantation embryos in 1981. ${ }^{1,2}$ Seventeen years later, human ESCs were also established from human blastocysts. ${ }^{3}$ Since then, several advances have been made in their application to regenerative medicine. In 2006, induced pluripotent stem cells (iPSCs), were generated from mouse embryonic and adult fibroblasts, namely from differentiated somatic cells. ${ }^{4}$ The following year, human iPSCs were also established from human differentiated somatic cells. ${ }^{5,6}$ iPSCs possess pluripotency and self-renewal properties similar to ESCs and have attracted attention as tools for regenerative medicine and drug development. 
Pluripotent ESCs and iPSCs are regulated by signaling from extrinsic factors as well as transcriptional control by intrinsic factors such as Oct3/4, Sox2, and Nanog. These factors maintain the cell's undifferentiated state or determine cell fate during the process of differentiation from PSCs. These functions involving differentiation are conserved among species and are exhibited by several common signals. Fibroblast growth factor (FGF) signal enhances the differentiation of PSCs into ectoderm and neural stem cells in mice and in humans. ${ }^{7,8}$ In FGF/extracellular signal-related kinases 1/2 (ERK1/2) signaling, FGF binds to its cognate receptor, after which ERK is phosphorylated and activated by mitogen-activated protein kinase (MAPK)/ERK kinase (MEK). Wnt signal is required for differentiation to mesoderm and endoderm in both mice and humans. ${ }^{9-11}$ In the canonical Wnt signaling pathway, Wnt binds to the cell surface receptors Frizzled and Lrp5/6, and then Dishevelled inhibits the phosphorylation of $\beta$-catenin via glycogen synthase kinase $3-\beta$. Stabilized cytoplasmic $\beta$-catenin translocates into the nucleus and works as a co-regulator for Tcf/Lef transcription factors, which in turn bind to target genes to modulate their expression. ${ }^{12}$

Recently, accumulating evidence has suggested that reactive oxygen species (ROS) regulate various signaling pathways in stem cells and differentiated cells. ${ }^{13-15}$ ROS are considered to be a possible cause for various diseases, including cancer and age-related illnesses. Three primary forms of intracellular ROS are the superoxide anion $\left(\mathrm{O}_{2}^{-}\right)$, hydrogen peroxide $\left(\mathrm{H}_{2} \mathrm{O}_{2}\right)$, and the hydroxyl radical $\left(\mathrm{OH}^{-}\right)$. Mitochondria and the nicotinamide adenine dinucleotide phosphate oxidase (NOX) family are the major sources of ROS in cells. ${ }^{16}$ For example, $\mathrm{O}^{2-}$ is generated by mitochondrial complex I \& III and NOX and is subsequently catalyzed by superoxide dismutase to $\mathrm{H}_{2} \mathrm{O}_{2}$, which has the longest half-life and can diffuse easily through membranes compared with other ROS forms. ${ }^{17}$ Therefore, $\mathrm{H}_{2} \mathrm{O}_{2}$ is considered to be the main ROS involved in intracellular signaling.

ESCs contain immature mitochondria, indicating reduced mitochondrial function. Therefore, ESCs depend on glycolysis rather than the mitochondrial oxidative phosphorylation pathway. ${ }^{13}$ ROS are considered to be lower in stem cells than in differentiated cells. However, ROS regulate various signaling pathways in ESCs and iPSCs. For instance, in mouse ESCs, ROS activate ERK1/2, Jun-N-terminal kinase (Jnk), and p38, which are the components of the MAPK signaling pathway. ${ }^{14,18,19}$ During nuclear reprogramming of iPSCs, ROS signaling is required in the early stages and then reduced in later stages. ${ }^{20} \mathrm{~A}$ large number of reactive species such as ROS and reactive nitrogen species (RNS) are generated by atmospheric pressure plasma (APP) irradiation in culture medium at room temperature. Therefore, APP irradiation has the potential to control the status of stem cells, namely self-renewal and differentiation. Here, we summarize the effects of plasma irradiation on mouse ESCs.

\section{EFECTOF APP IRRADIATION ON THE UNDIFERENTIATED STATE OF MOUSE ESCS}

APP-irradiated medium inhibits the proliferation of mouse ESCs, but does not affect their undifferentiated state; the expression level of the undifferentiated markers Oct3/4, 
Nanog, and Sox 2 did not change. ${ }^{21}$ We also found that the inhibition of mouse ESC proliferation induced by APP-irradiated medium was more substantial than that induced by direct APP irradiation. The results suggest that this effect is caused by $\mathrm{H}_{2} \mathrm{O}_{2}$ generated by APP irradiation in the culture medium.

\section{EFECTOF APP IRRADIATION ON DIFERENTIATION OF MOUSE ESCS}

\section{A. Embryoid Body Formation Mimics Embryo Differentiation}

Embryoid body (EB) formation is one of the experimental methods used to analyze the pluripotency of ESCs. ${ }^{22}$ This method mimics the in vivo differentiation of the mouse embryo into three germ layers, endoderm, mesoderm, and ectoderm, and is performed by culturing mouse ESCs in floating conditions without leukemia inhibitory factor, an essential factor for the maintenance of the undifferentiated state of mouse ESCs (Fig. 1A). Endoderm, mesoderm, and ectoderm differentiate into digestive organs, muscular tissues and blood cells, and neurons, respectively, in the adult. During EB formation, each germ layer marker is expressed along a time axis in the same manner as happens during early embryonic development (Fig. 1B). Mouse ESCs first differentiate into epiblasts, forming endodermal and mesodermal cells during the early stages (day 3 ), whereas the ectodermal cells differentiate, probably from epiblast cells, during the later stage (day 12). Fgf5, Foxa2, T, and Pax6 are representative markers for each developmental stage, namely epiblast, endoderm, mesoderm, and ectoderm, respectively. We used this in vitro differentiation system for the analysis of the effects of plasma irradiation.

\section{B. APP-Inadiated Medium Has More Substantial Effect on the Differentiation of Mouse ESCs than Direct APP Imadiation}

We prepared APP-irradiated medium as described previously ${ }^{23}$ and performed two investigations: (1) EB formation was evaluated in APP-irradiated medium for 12 days after direct APP irradiation, (2) EB formation was evaluated in APP-irradiated medium without direct APP-irradiation. Then, we analyzed the marker of each developmental stage: epiblast, endoderm, mesoderm, and ectoderm. In both experiments, epiblast marker and ectodermal marker levels increased, whereas the mesodermal marker level decreased (Fig. 2). No significant difference could be observed between the two investigations, indicating that APP-irradiated medium has a more substantial effect on differentiation of mouse ESCs than direct APP irradiation.

\section{APP-Inadiated Medium Has No Effect on the Proliferation of Bs}

APP-irradiated medium contains $\mathrm{H}_{2} \mathrm{O}_{2}$, one of the main reactive species generated by plasma irradiation. ${ }^{24}$ Subsequently, we detected $45 \mu \mathrm{M} \mathrm{H}_{2} \mathrm{O}_{2}$ in ultrapure water (Wako) after $60 \mathrm{sec}$ of APP irradiation using the same conditions as those used in the preparation of APP-irradiated medium. ${ }^{21,23}$ Under these conditions, the $\mathrm{pH}$ of the medium was unchanged 
A

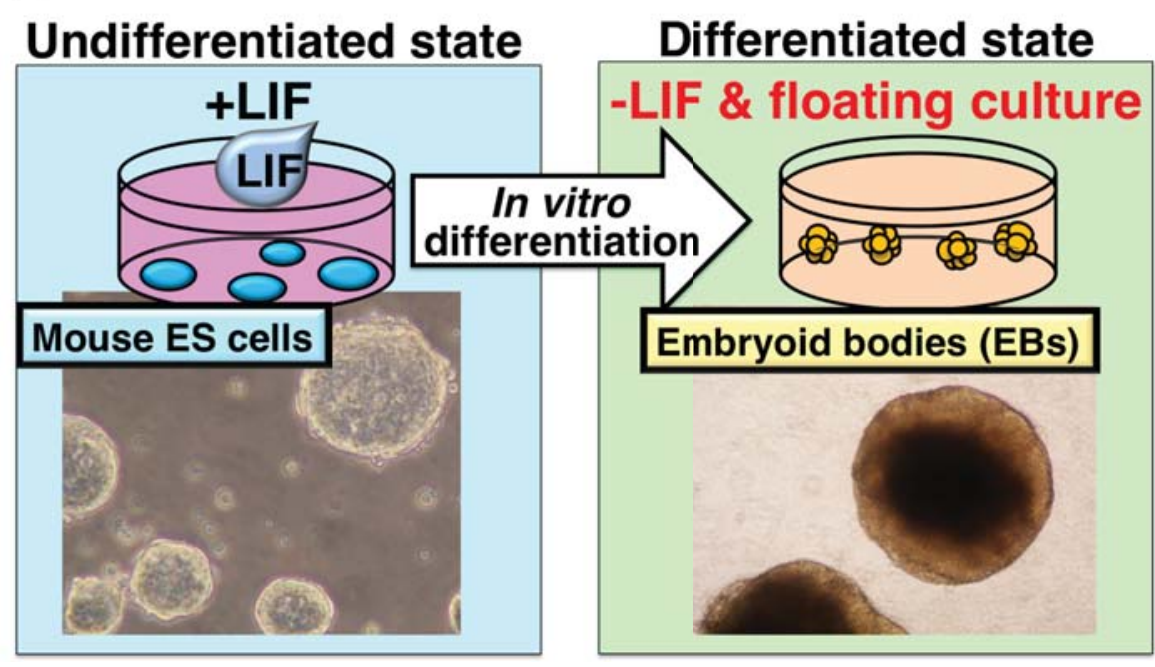

\section{B}

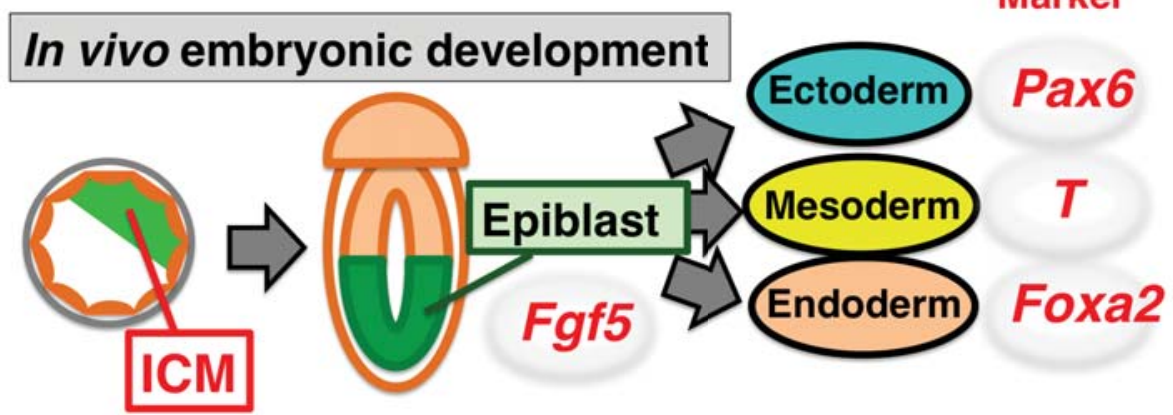

\section{In vitro EB formation}

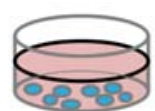

ESCs

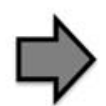

Epiblast

Day 2.5 Fgf5
Day 12

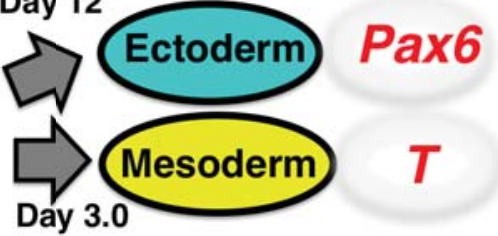

5

FIG. 1: EB formation mimics in vivo differentiation of mouse embryo. ${ }^{22}$ (A) Floating culture of ESCs without leukemia inhibitory factor (LIF), an essential factor for the maintenance of the undifferentiated state of mouse ESCs, induces EB differentiation to produce three germ layers: endoderm, mesoderm, and ectoderm. (B) During EB formation, each germ layer marker is expressed along a time axis in the same manner as early embryonic development. The epiblast marker Fgf5 is up-regulated on day 2.5. The endodermal marker Foxa2 and the mesodermal marker T are up-regulated on day 3. The ectodermal marker Pax6 is upregulated on day 12. 


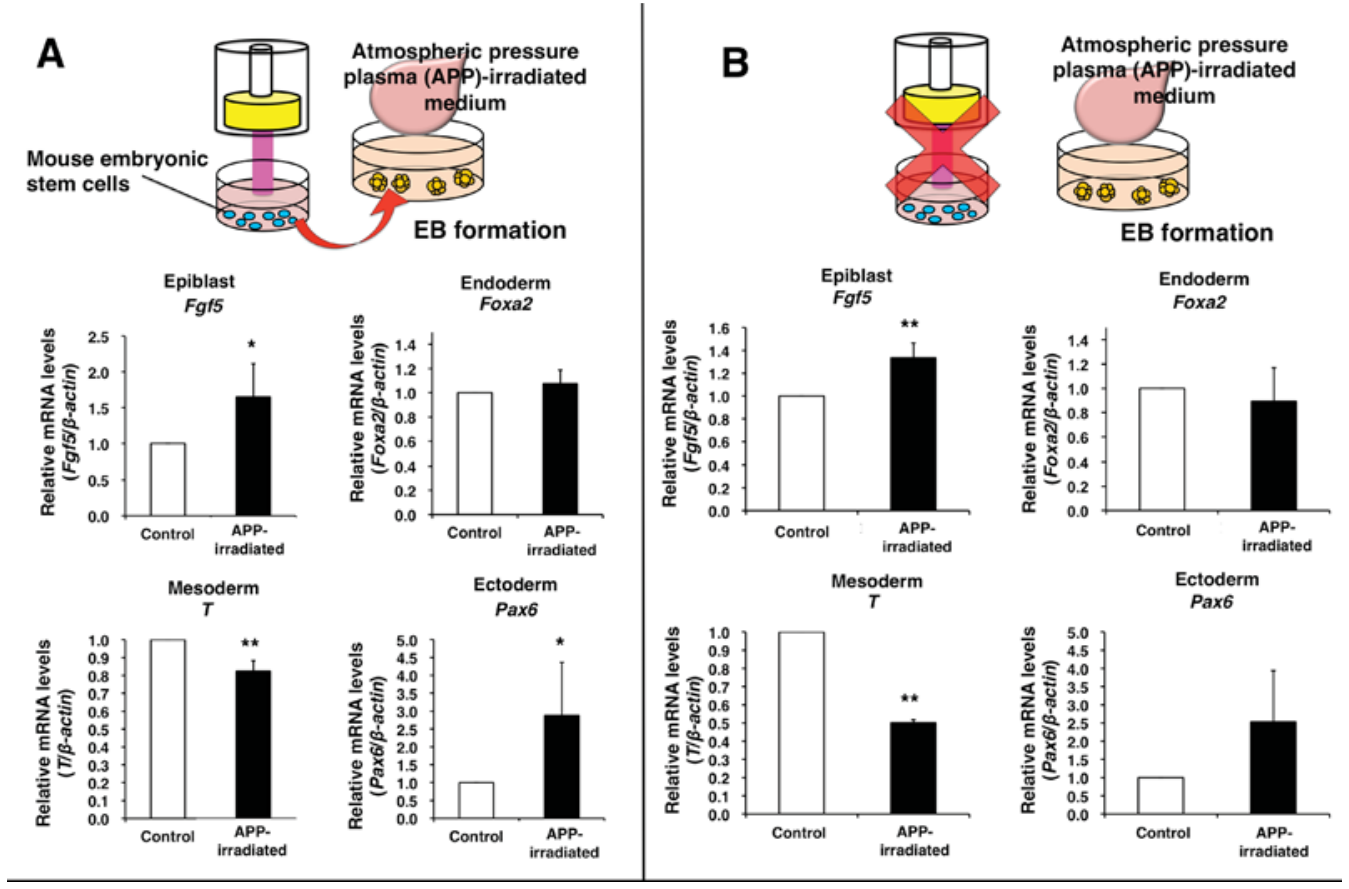

FIG. 2: APP-irradiated medium has a more substantial effect on differentiation of mouse ESCs than direct APP irradiation. The relative amounts of mRNA are shown after normalization against controls (value $=1$ ). The values shown are the mean \pm standard deviation $(\mathrm{SD})$ of three independent experiments; ${ }^{*} \mathrm{p}<0.05 ; * \mathrm{*}<0.01$ compared with controls. (A) After direct APP irradiation, we evaluated EB formation in APP-irradiated medium. Subsequently, we analyzed the markers of epiblast, endoderm, and mesoderm on day 4 and that of ectoderm on day 12 by real-time polymerase chain reaction (PCR). (B) Without direct APP irradiation, we evaluated EB formation in APP-irradiated medium and analyzed the markers of epiblast, endoderm, and mesoderm on day 4 and that of ectoderm on day 12 by real-time PCR.

by APP irradiation. To determine whether the effects of plasma irradiation were caused by the main reactive species, $\mathrm{H}_{2} \mathrm{O}_{2}$, or by other irradiation-generated species, we prepared $\mathrm{H}_{2} \mathrm{O}_{2}$-supplemented, non-irradiated medium and APP-irradiated medium and evaluated both for EB formation. The morphology and size of EBs cultured in APP-irradiated medium and $\mathrm{H}_{2} \mathrm{O}_{2}$-supplemented, non-irradiated medium did not change compared with controls, ${ }^{23}$ indicating that APP irradiation and $\mathrm{H}_{2} \mathrm{O}_{2}$ did not affect the proliferation of EBs, whereas mouse ESC proliferation was inhibited by APP-irradiated medium. ${ }^{21}$

\section{APP-Inradiated Medium Enhances Epiblast Differentiation by Activating FGF4 Signaling}

During EB formation, mouse ESCs differentiate first into epiblast and then into all three germ layers. ${ }^{22}$ The effects of APP-irradiated medium and $\mathrm{H}_{2} \mathrm{O}_{2}$-supplemented, non-irra- 
diated medium on the differentiation to epiblast were analyzed in EBs on day 2.5. ${ }^{23} \mathrm{In}$ both media, the level of epiblast markers Fgf5 and Otx2 increased, indicating that differentiation into epiblasts was enhanced by both APP irradiation and $\mathrm{H}_{2} \mathrm{O}_{2}$.

FGF4 signaling works as a trigger for the differentiation into epiblasts. ${ }^{25,26}$ Fas signaling also enhances the differentiation into epiblasts independently of FGF4 signaling. ${ }^{27}$ In both media, phosphorylation of Erk1/2, one of the downstream components of FGF4 signaling, was significantly increased and the expression levels of Spry2 and Dusp6, target genes of FGF4 signaling, were up-regulated. ${ }^{23}$ However, the expression of cleaved Caspase 3 did not change. This indicates that FGF4 signaling is enhanced in APP-irradiated medium due to the $\mathrm{H}_{2} \mathrm{O}_{2}$ generated by APP irradiation.

FGF4 binds to FGF receptor 2c to activate FGF4 signaling. ${ }^{26} \mathrm{Fgf4}$ and $\mathrm{Fgfr} 2 \mathrm{c}$ mRNA increased in both media, demonstrating that the enhancement of FGF4 signaling was caused by the up-regulation of FGF4 and $F g f r 2 c .{ }^{23}$ Therefore, APP irradiation, or rather $\mathrm{H}_{2} \mathrm{O}_{2}$ generated by APP irradiation, promoted the expression of $F G F 4$ and $F g f r 2 c$, up-regulated FGF4 signaling, and then enhanced epiblast differentiation in EBs on day 2.5.

\section{E. APP-Inadiated Medium Inhibited Endodemal and Mesodemal Differentiations by Inhibiting Wnt Signaling}

Epiblast cells differentiate into endodermal or mesodermal cells, with the differentiation into ectodermal cells beginning later. ${ }^{22}$ The effects of APP-irradiated medium and $\mathrm{H}_{2} \mathrm{O}_{2}$-supplemented, non-irradiated medium on the differentiation to endoderm or mesoderm were analyzed in EBs on day $3 .^{23}$ The level of the endodermal markers Foxa 2 and Gata6 was decreased in APP-irradiated medium, whereas it was unchanged in $\mathrm{H}_{2} \mathrm{O}_{2}$-supplemented medium. The level of the mesodermal markers $T$ and Mixll was decreased in APP-irradiated medium, whereas the level increased in $\mathrm{H}_{2} \mathrm{O}_{2}$-supplemented medium. This demonstrates that the inhibition of endodermal and mesodermal differentiation is a specific effect of APP irradiation rather than of the generated $\mathrm{H}_{2} \mathrm{O}_{2}$.

Wnt signaling enhances the differentiation to endoderm and mesoderm. ${ }^{28}$ The expression of the Wnt signaling target genes Axin2 and CcnD1 was decreased in APPirradiated medium and unchanged in $\mathrm{H}_{2} \mathrm{O}_{2}$-supplemented medium. Wnt3a is a Wnt ligand, regulating endodermal and mesodermal differentiation. ${ }^{29}$ Wnt3a binds to the receptors Lrp5 or Lrp6, as well as to Frizzled1 or Frizzled5, after which the downstream components of the Wnt signaling pathway are activated. Expression levels of all receptors were decreased in the APP-irradiated medium, but unchanged in $\mathrm{H}_{2} \mathrm{O}_{2}$ supplemented medium, whereas Wnt3a was unchanged in either medium. ${ }^{23}$ Therefore, on day 3, APP-irradiated medium inhibited Wnt3a receptors, suppressed Wnt signaling, and consequently inhibited endodermal and mesodermal differentiation in EBs. $\mathrm{H}_{2} \mathrm{O}_{2}$ generated by APP irradiation did not cause this effect. 


\section{F. APP-Inadiated Medium Enhances Ec todemal Differentiation and Subsequent Neuronal Differentiation by Activating FGF Signaling}

The effects of APP-irradiated medium and $\mathrm{H}_{2} \mathrm{O}_{2}$-supplemented, non-irradiated medium on the differentiation into ectoderm and subsequent differentiation to neuronal lineages were analyzed on day $12 .{ }^{23}$ In both media, the ectoderm markers Pax6 and Nestin were up-regulated, as was the neural marker Tubb3, whereas the astrocyte marker GFAP and the oligodendrocyte marker Olig1 were unchanged. This indicates that the differentiation into ectoderm and subsequently to neurons was enhanced by both APP irradiation and $\mathrm{H}_{2} \mathrm{O}_{2}$. These results are consistent with a previous study stating that plasma irradiation induces selective neuronal differentiation of neural stem cells. ${ }^{30}$

FGF4 signaling enhances ectodermal differentiation and subsequent neuronal differentiation. ${ }^{7}$ In both media, expressions levels of FGF4 signaling target genes Spry2 and Dusp6 were up-regulated. ${ }^{23} F g f 4$ and $F g f r 2 c$ mRNA levels increased in both media, indicating that $F G F 4$ and $F g f r 2 c$ expression was enhanced by $\mathrm{H}_{2} \mathrm{O}_{2}$ generated by APP irradiation. Therefore, in EBs on day 12, APP irradiation, or rather $\mathrm{H}_{2} \mathrm{O}_{2}$ generated by APP irradiation, promoted the expression of FGF4 and Fgfr $2 c$, up-regulated FGF4 signaling, and then enhanced ectodermal and neuronal differentiation in EBs.

\section{G. RNS Did Not Inhibit Endodemal and Mesodemal Differentiation}

As mentioned in section III-E, "APP-Irradiated Medium Inhibited Endodermal and Mesodermal Differentiations by Inhibiting Wnt Signaling," the inhibition of endodermal and mesodermal differentiation was a specific effect of APP irradiation and was not induced by the generated $\mathrm{H}_{2} \mathrm{O}_{2}$. In addition to ROS such as $\mathrm{H}_{2} \mathrm{O}_{2}$, RNS including NO, $\mathrm{NO}_{2}^{-}$, and $\mathrm{NO}_{3}^{-}$are generated by plasma irradiation in the culture medium. Therefore, we analyzed the effect of RNS on endodermal and mesodermal differentiation.

At first, we determined the concentration of RNS after $60 \mathrm{sec}$ of APP irradiation on ultrapure water (Wako) using the same conditions as those used in the preparation of APP-irradiated medium. ${ }^{23}$ Generated $\mathrm{NO}$ is oxidized to $\mathrm{NO}_{2}^{-}$or $\mathrm{NO}_{3}^{-}$in liquid form. The total concentration of $\mathrm{NO}_{2}{ }^{-}$and $\mathrm{NO}_{3}{ }^{-}$was determined by using Griess reagent kit (Dojindo) according to the manufacturer's instructions. ${ }^{31}$ In total, $2 \mu \mathrm{M} \mathrm{NO}_{2}{ }^{-}$and $\mathrm{NO}_{3}{ }^{-}$ was generated in APP-irradiated ultrapure water.

Diethylenetriamine nitric oxide (DETA-NO) is an NO donor that is often used to release $\mathrm{NO}$ in the culture medium ${ }^{32}$ in a process in which 2 moles of $\mathrm{NO}$ are dissociated from 1 mole of DETA-NO in the culture medium. Therefore, 2 moles of $\mathrm{NO}_{2}^{-}$or $\mathrm{NO}_{3}^{-}$ are produced from 1 mole of DETA-NO in the medium. We evaluated EB formation using $1 \mu \mathrm{M}$ DETA-NO (Sigma-Aldrich) for 3 days to analyze the effect of $\mathrm{NO}_{2}^{-}$and $\mathrm{NO}_{3}^{-}$on endodermal and mesodermal differentiations. DETA-NO did not affect the morphology or size of EBs (Fig. 3), demonstrating that $\mathrm{NO}_{2}{ }^{-}$and $\mathrm{NO}_{3}{ }^{-}$did not affect the proliferation of EBs.

Finally, the expressions levels of endodermal markers Foxa2 and Gata6 and the mesodermal markers $T$ and Mixll were analyzed in EBs on day 3 by real-time poly-

Volume 7, Issue 3, 2017 


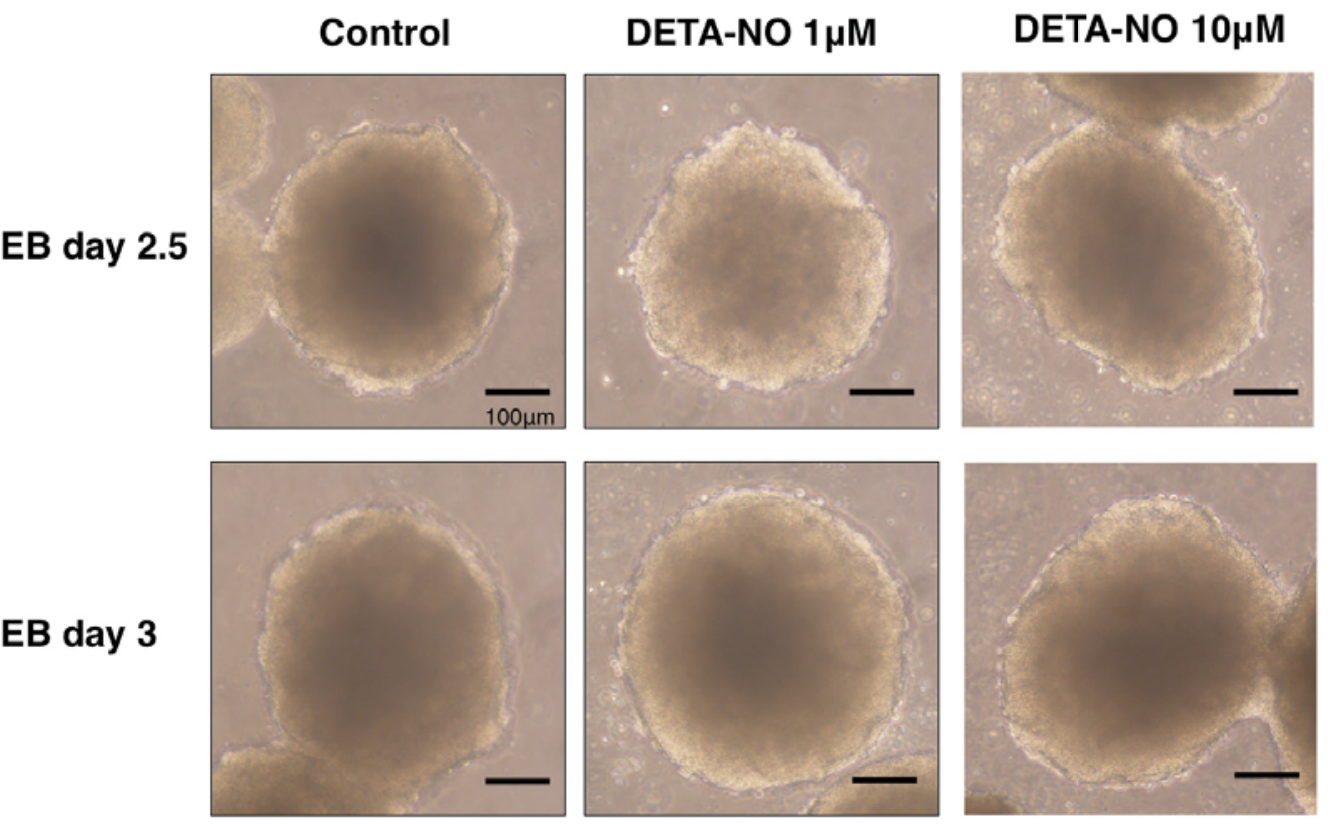

FIG. 3: RNS $\left(\mathrm{NO}_{2}^{-}\right.$and $\left.\mathrm{NO}_{3}^{-}\right)$do not affect the proliferation of EBs. EB formation was evaluated in cultured medium with and without DETA-NO. Photomicrographs show EBs on day 2.5 and 3 after EB formation. The scale bar indicates $100 \mu \mathrm{m}$.

merase chain reaction. Levels of all markers increased in EBs cultured in medium with $1 \mu \mathrm{M}$ DETA-NO compared with control. DETA-NO enhanced the differentiation to endoderm and mesoderm, demonstrating that $\mathrm{NO}_{2}{ }^{-}$and $\mathrm{NO}_{3}{ }^{-}$promoted endodermal and mesodermal differentiation (Fig. 4). Similar results were obtained in EBs cultured in medium with $10 \mu \mathrm{M}$ DETA-NO. Therefore, it is clear that the inhibition of differentiation to endoderm and mesoderm by APP irradiation was not caused by $\mathrm{NO}_{2}^{-}$or $\mathrm{NO}_{3}^{-}$.

\section{CONCLUSION}

Here, we summarize the effects of plasma irradiation on the maintenance and differentiation of mouse ESCs. APP irradiation inhibited the proliferation of mouse ESCs, but did not affect their undifferentiated state. In contrast, plasma irradiation did not inhibit the proliferation of EBs, but inhibited the differentiation from mouse ESCs to endoderm and mesoderm by inhibiting Wnt signal (Fig. 5). These effects were not caused by $\mathrm{H}_{2} \mathrm{O}_{2}$, $\mathrm{NO}_{2}^{-}$, or $\mathrm{NO}_{3}^{-}$, indicating that some other factors specific to APP irradiation may be involved. Moreover, APP irradiation enhanced the differentiation to the epiblast, ectoderm, and neuronal lineages by activation of FGF4 signaling brought about by the $\mathrm{H}_{2} \mathrm{O}_{2}$ generated by APP irradiation in the culture medium. 

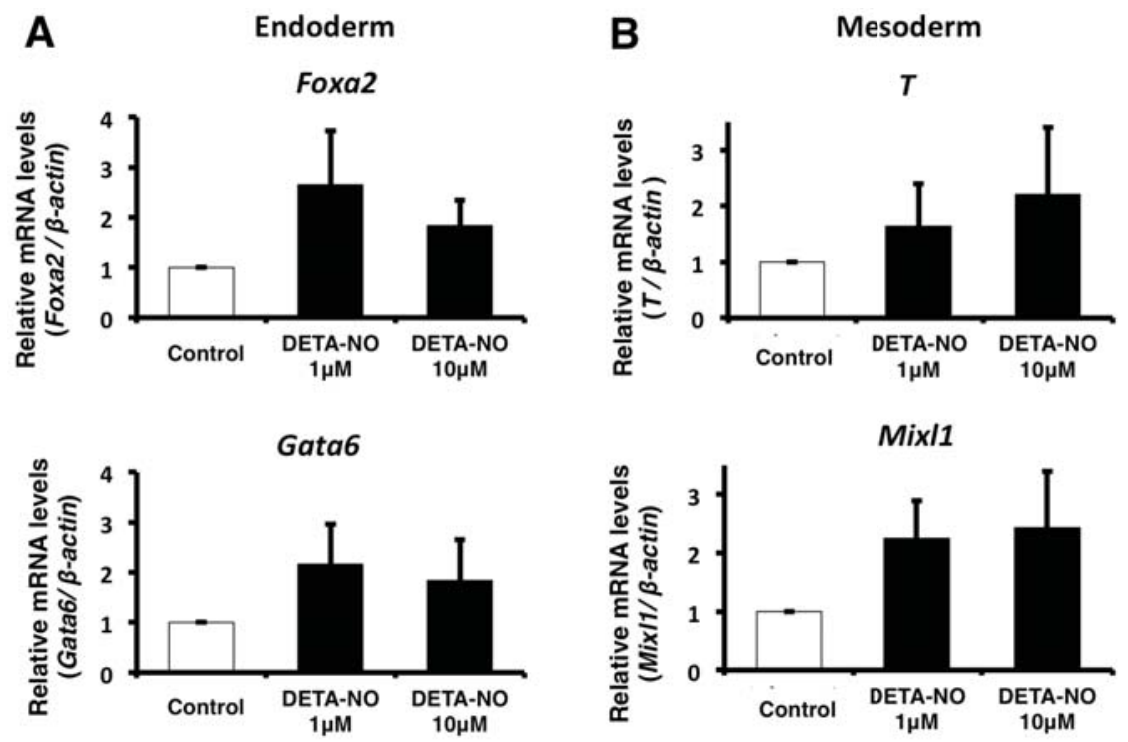

FIG. 4: $\mathrm{RNS}\left(\mathrm{NO}_{2}^{-}\right.$and $\left.\mathrm{NO}_{3}^{-}\right)$enhance differentiation into endoderm and mesoderm. EB formation was evaluated in cultured medium with and without DETA-NO. The endodermal (A) and mesodermal (B) markers were analyzed on day 3 by real-time PCR. The relative amounts of mRNA are shown after normalization against controls (value $=1$ ). The values shown are the means \pm standard deviation (SD) of three independent experiments.

\section{Differentiation from ESCs}

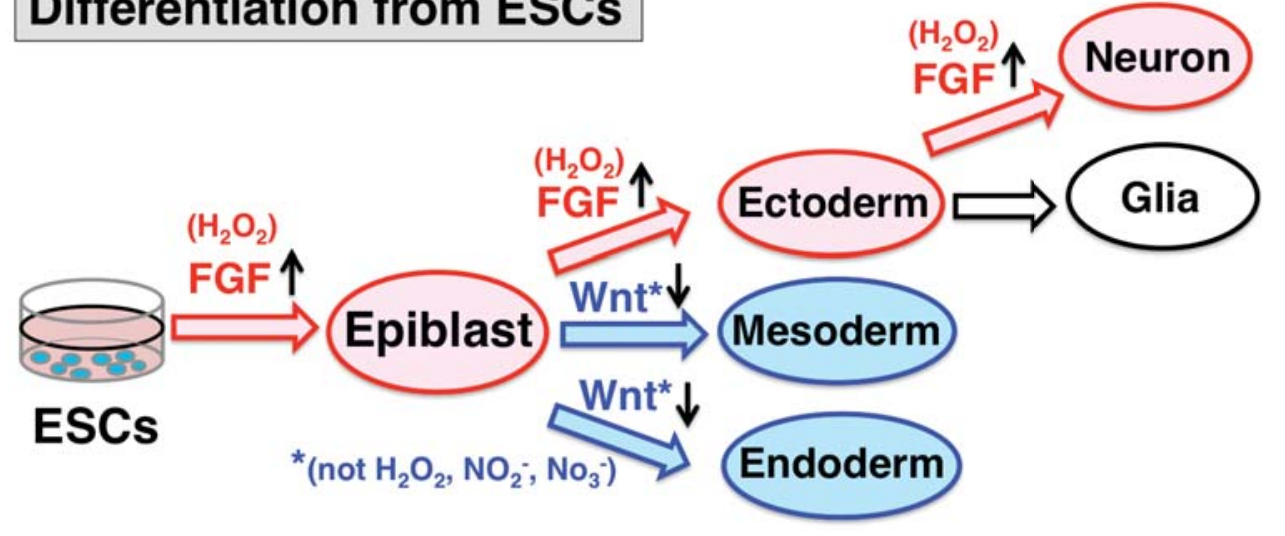

FIG. 5: Schematic representation of the effects of APP irradiation on the differentiation of mouse ESCs. Red (lighter) and blue (darker) indicate "up-regulated" and "down-regulated" paths or components, respectively. APP irradiation enhanced the differentiation of mouse ESCs into epiblast, ectodermal, and neuronal lineages by activation of FGF4 signaling brought about by $\mathrm{H}_{2} \mathrm{O}_{2}$ generated by APP irradiation in the medium. In contrast, APP irradiation inhibited differentiation into mesoderm and endoderm by inhibiting Wnt signaling. $\mathrm{H}_{2} \mathrm{O}_{2}, \mathrm{NO}_{2}^{-}$, and $\mathrm{NO}_{3}^{-}$were not responsible for this effect, indicating that some other factors specific to APP irradiation may be involved. 


\section{ACKNOWFDGMENT}

This work was supported in part by a Grant-in-Aid for Scientific Research on Innovative Area 15H00904 from the Ministry of Education, Culture, Sports, and Science of Technology (MEXT) of Japan.

\section{REFERENCES}

1. Evans MJ, Kaufman MH. Establishment in culture of pluripotential cells from mouse embryos. Nature. 1981;292:154-6.

2. Martin GR. Isolation of a pluripotent cell line from early mouse embryos cultured in medium conditioned by teratocarcinoma stem cells. Proc Natl Acad Sci U S A. 1981;78:7634-8.

3. Thomson JA, Itskovitz-Eldor J, Shapiro SS, Waknitz MA, Swiergiel JJ, Marshall VS, Jones JM. Embryonic stem cell lines derived from human blastocysts. Science. 1998;282:1145-7.

4. Takahashi K, Yamanaka S. Induction of pluripotent stem cells from mouse embryonic and adult fibroblast cultures by defined factors. Cell. 2006;126:663-76.

5. Takahashi K, Tanabe K, Ohnuki M, Narita M, Ichisaka T, Tomoda K, Yamanaka S. Induction of pluripotent stem cells from adult human fibroblasts by defined factors. Cell. 2007;131:861-72.

6. Yu J, Vodyanik MA, Smuga-Otto K, Antosiewicz-Bourget J, Frane JL, Tian S, Nie J, Jonsdottir GA, Ruotti V, Stewart R, Slukvin II Thomson JA. Induced pluripotent stem cell lines derived from human somatic cells. Science. 2007;318:1917-20.

7. Kosaka N, Kodama M, Sasaki H, Yamamoto Y, Takeshita F, Takahama Y, Sakamoto H, Kato T, Terada M, Ochiya T. FGF-4 regulates neural progenitor cell proliferation and neuronal differentiation. FASEB J. 2006;20:1484-5.

8. Chen SM, Lee MS, Chang CY, Lin SZ, Cheng EH, Liu YH, Pan HC, Lee HC, Su HL. Prerequisite OCT4 maintenance potentiates the neural induction of differentiating human embryonic stem cells and induced pluripotent stem cells. Cell Transplant. 2015;24:829-44.

9. Lindsley RC, Gill JG, Kyba M, Murphy TL, Murphy KM. Canonical Wnt signaling is required for development of embryonic stem cell-derived mesoderm. Development. 2006;133:3787-96.

10. Loh KM, Ang LT, Zhang J, Kumar V, Ang J, Auyeong JQ, Lee KL, Choo SH, Lim CY, Nichane M, Tan J, Noghabi MS, Azzola L, Ng ES, Durruthy-Durruthy J, Sebastiano V, Poellinger L, Elefanty AG, Stanley EG, Chen Q, Prabhakar S, Weissman IL, Lim B. Efficient endoderm induction from human pluripotent stem cells by logically directing signals controlling lineage bifurcations. Cell Stem Cell. 2014;14:237-52.

11. Price FD, Yin H, Jones A, Van Ijcken W, Grosveld F, Rudnicki MA. Canonical Wnt signaling induces a primitive endoderm metastable state in mouse embryonic stem cells. Stem Cells. 2013;31:752-64.

12. MacDonald BT, Tamai K, He X. Wnt/ $\beta$-catenin signaling: Components, mechanisms, and diseases. Dev Cell. 2009;17:9-26.

13. Bigarella CL, Liang R, Ghaffari S. Stem cells and the impact of ROS signaling. Development. 2014;141:4206-18.

14. Ren F, Wang K, Zhang T, Jiang J, Nice EC, Huang C. New insights into redox regulation of stem cell self-renewal and differentiation. Biochim Biophys Acta. 2015;1850:1518-26.

15. Chaudhari P, Ye Z, Jang YY. Roles of reactive oxygen species in the fate of stem cells. Antioxid Redox Signal. 2014;20:1881-90.

16. Tahara EB, Navarete FDT, Kowaltowski AJ. Tissue-, substrate-, and site-specific characteristics of mitochondrial reactive oxygen species generation. Free Radic Biol Med. 2009;46:1283-97.

17. Holmström KM, Finkel T. Cellular mechanisms and physiological consequences of redox-dependent signalling. Nat Rev Mol Cell Biol. 2014;15:411-21.

18. Sauer H, Wartenberg M. Reactive oxygen species as signaling molecules in cardiovascular differentia- 
tion of embryonic stem cells and tumor-induced angiogenesis. Antioxid Redox Signal. 2005;7:142334.

19. Valko M, Leibfritz D, Moncol J, Cronin M T, Mazur M, Telser J. Free radicals and antioxidants in normal physiological functions and human disease. Int J Biochem Cell Biol. 2007;39:44-84.

20. Zhou G, Meng S, Li Y, Ghebre YT, Cooke JP. Optimal ROS signaling is critical for nuclear reprogramming. Cell Rep. 2016;15:919-25.

21. Miura T, Ando A, Hirano K, Ogura C, Kanazawa T, Ikeguchi M, Seki A, Nishihara S, Hamaguchi S. Proliferation assay of mouse embryonic stem (ES) cells exposed to atmospheric-pressure plasmas at room temperature. J Phys D Appl Phys. 2014;47:445402.

22. Jackson M, Taylor AH, Jones EA, Forrester LM. The culture of mouse embryonic stem cells and formation of embryoid bodies. Methods Mol Biol. 2010;633:1-18.

23. Miura T, Hamaguchi S, Nishihara S. Atmospheric-pressure plasma-irradiation inhibits mouse embryonic stem cell differentiation to mesoderm and endoderm but promotes ectoderm differentiation. J Phys D Appl Phys. 2016;49:165401.

24. Winter J, Tresp H, Hammer MU, Iséni S, Kupsch S, Schmidt-Bleker A, Wende K, Dünnbier M, Masur $\mathrm{K}$, Weltmann KD, Reuter $\mathrm{S}$. Tracking plasma generated $\mathrm{H} 2 \mathrm{O} 2$ from gas into liquid phase and revealing its dominant impact on human skin cells. J Phys D Appl Phys. 2014;47:285401.

25. Kunath T, Saba-El-Leil M K, Almousailleakh M, Wray J, Meloche S, Smith A. FGF stimulation of the Erk1/2 signalling cascade triggers transition of pluripotent embryonic stem cells from self-renewal to lineage commitment. Development. 2007;134:2895-902.

26. Lanner F, Rossant J. The role of FGF/Erk signaling in pluripotent cells. Development. 2010;137:335160.

27. Hirano K, Sasaki N, Ichimiya T, Miura T, Kuppevelt TH, Nishihara S. 3-O-sulfated heparan sulfate recognized by the antibody HS4C 3 contributes to the differentiation of mouse embryonic stem cells via Fas signaling. PLoS One. 2012;7:e43440.

28. Price FD, Yin H, Jones A, Van Ijcken W, Grosveld F, Rudnicki MA. Canonical Wnt signaling induces a primitive endoderm metastable state in mouse embryonic stem cells. Stem Cells. 2013;31:752-64.

29. Lien WH, Fuchs E. Wnt some lose some: transcriptional governance of stem cells by Wnt/ $\beta$-catenin signaling. Genes Dev. 2014:28;1517-32.

30. Xiong Z, Zhao S, Mao X, Lu X, He G, Yang G, Chen M, Ishaq M, Ostrikov K. Selective neuronal differentiation of neural stem cells induced by nanosecond microplasma agitation. Stem Cell Res. 2013;12:387-99.

31. Muratsu D, Yoshiga D, Taketomi T, Onimura T, Seki Y, Matsumoto A, Nakamura S. Zoledronic acid enhances lipopolysaccharide-stimulated proinflammatory reactions through controlled expression of SOCS1 in macrophages. PLoS One. 2013;8:e67906.

32. Mora-Castilla S, Tejedo JR, Hmadcha A, Cahuana GM, Martín F, Soria B, Bedoya FJ. Nitric oxide repression of Nanog promotes mouse embryonic stem cell differentiation. Cell Death Differ. 2010;17:1025-33.

Volume 7, Issue 3, 2017 
